\title{
Irreducible homomorphisms for lattices over orders
}

\author{
Joachim W. Schmidt
}

\begin{abstract}
Let $\Lambda$ be a complete $R$-order in the semi-simple $K$-algebra $A$. Then it has been shown that for each indecomposable $\Lambda$-lattice $M \quad$ which is not projective, there exists a unique almost split sequence $0 \rightarrow N \rightarrow E \rightarrow M \rightarrow 0$. Here we study the middle term $E$ and characterize those almost split sequences where $E$ has a projective direct summand. In the case where $\Lambda$ is the groupring $R G$ for a finite group $G$, we get information about the almost split sequences for the syzygies and apply our results in an example.
\end{abstract}

\section{0 . Introduction}

Throughout this paper $K$ is the $p$-adic completion of an algebraic number field, $R$ is the ring of integers in $K$ and $\Lambda$ is an $R$-order in a finite dimensional semi-simple $K$-algebra $A$. By $\Lambda^{\mathrm{M}}$ we denote the category of left $\Lambda$-lattices, that is, finitely generated left modules which are $R$-projective.

In [6] and [2] it, ras been shown that for this situation there exist 'almost split sequences'. This means that for each indecomposable nonprojective $M \in \stackrel{M^{0}}{=}$ there exists a unique short exact sequence of $\Lambda$-lattices

$$
\underline{\underline{\mathrm{G}}}: 0 \rightarrow N \stackrel{\varphi}{\longrightarrow} E \stackrel{\psi}{\longrightarrow} M \rightarrow 0
$$

Received 21 February 1977. 
with the following property: $\underline{\underline{G}}$ does not split, $N$ is indecomposable, and for each $\alpha \in \operatorname{hom}_{\Lambda}(X, M)$ which is not a splitable epimorphism, there exists $\beta \in \operatorname{hom}_{\Lambda}(X, E)$ such that $\beta \psi=\alpha$. Auslander has shown $[1,3]$ that the study of almost split sequences for finitely generated modules over Artin algebras leads to the concept of irreducible morphisms.

Using this idea for our situation, we obtain much information about almost split sequences over orders, especially about the middle term $E$. This we present in $\$ 1 . \$ 2$ is devoted to study in more detail the special class of Gorenstein orders, which includes group-rings $R G$ for finite groups $G$. So we get the following result.

If the middle term $E$ of an almost split sequence $G$ has a projective direct summand $P$, then $E=P \oplus E_{1}$ with $\operatorname{rank}(N)=\operatorname{rk}\left(E_{1}\right)=\operatorname{rk}(M)=\operatorname{rk}(P)$ and $P$ is indecomposable. Another result says that in the almost split sequences for the syzygies of a finite group the middle terms are indecomposable. In the last section, 3 , we study the group-ring $R G$ for the cyclic group $G$ of order $p^{2}$ in order to apply our previous results.

Concerning our notation, we remark that 'ring' means ring with identity and 'module' means unitary left-module. The composition $\varphi \psi$ of morphisms means that $\varphi$ is taken first. Finally we assume that all orders under consideration are not hereditary, since there the notion of almost split sequences makes no sense.

\section{Irreducible homomorphisms}

We start with the following definition (compare $[1,3]$ ).

DEFINITION 1.1. $\varphi \in \operatorname{hom}_{\Lambda}(X, Y)$ is said to be irreducible, if the following holds:

(a) $\varphi$ is neither a splitting epimorphism nor a splitting monomorphism;

(b) the factorization $\varphi=\alpha \beta$ with $\alpha \in \operatorname{hom}_{\Lambda}(X, 2)$, $B \in \operatorname{hom}_{\Lambda}(Z, Y)$ implies that $\alpha$ is a splittable monomorphism or $\beta$ is a splittable epimorphism. 
Now we list some of the basic properties of irreducible homomorphisms.

PROPOSITION 1.2. Let $\varphi \in \operatorname{hom}_{\Lambda}(X, Y)$ be irreducible. Then

(a) $\varphi$ is either a proper monomorphism or a proper epimorphism;

(b) if $\varphi$ is a monomorphism, then $\operatorname{cok}(\varphi)$ is an indecomposable $\Lambda$-module and if $\varphi$ is an epimorphism, then $\operatorname{ker}(\varphi)$ is an indecomposable $\Lambda$-lattice;

(c) suppose $\varphi$ is a monomorphism; then $\varphi$ is either a pure monomorphism, that is $\operatorname{cok}(\varphi)$ is a $\Lambda$-lattice, or $\varphi$ is a monomorphism onto a maximal submodule of $Y$.

Proof. (a) and (b) do not depend on the category $\Lambda \stackrel{M}{ }^{0}$ and can be proven straightforwardly (compare $[1,3]$ ).

(c). We have the exact sequence $0 \rightarrow X \stackrel{\varphi}{\rightarrow} Y \rightarrow Y / \operatorname{Im} \varphi \rightarrow 0$. Assume that $Y / \operatorname{Im} \varphi$ is not a lattice. Then there exists a $z \in \Lambda^{0}$ with $\operatorname{Im} \varphi \underset{+}{\subset} Z \subset Y$, such that $Z / \operatorname{Im} \varphi$ is the torsion part of $Y / \operatorname{Im} \varphi$. Thus we obtain a factorization of $\varphi$ via $Z$,

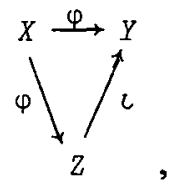

where $\iota$ denotes the inclusion. Since $Z / \operatorname{Im} \varphi$ is not a lattice, $\varphi: X \rightarrow Z$ cannot be a splittable monomorphism, which implies that $\iota: Z \rightarrow Y$ is a splittable epimorphism and so $Z=Y$. Thus $Y / \operatorname{Im} \varphi$ is a torsion module. From this we conclude that $\operatorname{Im} \varphi$ must be maximal in $Y$, since otherwise there would exist a $T: \operatorname{Im} \varphi \subsetneq T \subsetneq Y$; then the factorization of $\varphi$ via $T \underset{+}{\subset} Y$,

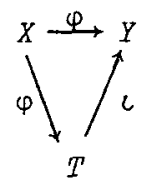

would imply that $\varphi: X \rightarrow T$ is a splittable monomorphism and so $T / \operatorname{Im} \varphi \neq 0$ 
would be torsionfree as a submodule of the torsion module $Y / \operatorname{Im} \varphi$, a contradiction. //

The reader should know that both cases of Proposition 1.2 (c) actually occur, as we shall show later on:

Now we describe the connection between irreducible morphisms and an almost split sequence $0 \rightarrow N \stackrel{\varphi}{\rightarrow} E \stackrel{\psi}{\longrightarrow} M \rightarrow 0$.

THEOREM 1.3. (a) If $\rho \in \operatorname{hom}_{\Lambda}(X, M)$, respectively $n \in \operatorname{hom}_{\Lambda}(N, Y)$, are irreducible, then $X$, respectively $Y$, are isomorphic to direct summands of $E$.

(b) If $E=\bigoplus_{i=1}^{n} E_{i}$ is a decomposition of the middle term $E$ with injections $\left\{c_{i}\right\}$ and projections $\left\{\pi_{i}\right\}$, then $\varphi \pi_{i}$ and $\iota_{i} \psi$ are irreducible for each $i \in\{1, \ldots, n\}$.

Proof. (a) Let $\rho \in \operatorname{hom}_{\Lambda}(X, M)$ be irreducible. Then $\rho$ is not a splittable epimorphism and the factorization property for an almost split sequence gives rise to $\mu \in \operatorname{hom}_{\Lambda}(X, E)$ with $\mu \psi=\rho$. Since $\psi$ is not a splittable epimorphism, $\mu$ must be asplittable monomorphism, which means that $X$ is isomorphic to a direct summand of $E$. Since for almost split sequences there holds a dual factorization property [7], the second part of (a) can be shown in an analogous way.

(b) This part is not as obvious as the above one. We are using the machinery of functor categories, which has enabled us to show the existence of almost split sequences [7]. Let $\iota_{i} \psi \in \operatorname{hom}_{\Lambda}\left(E_{i}, M\right) ; \iota_{i} \psi$ is neither a splittable monomorphism nor a splittable epimorphism, since otherwise the almost split sequence would split. Assume now that we have the commutative diagram

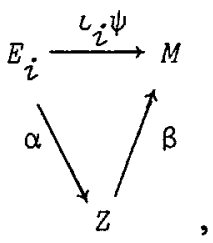

where $\beta$ is not asplittable epimorphism. Then we have to show that $\alpha$ is 
asplittable monomorphism. $\beta \in \operatorname{hom}_{\Lambda}(Z, M)$ induces a morphism $\beta_{*}$ in the category of additive contravariant functors from $\Lambda^{\circ}$ to abelian groups, namely $\beta_{*}: \operatorname{hom}_{\Lambda}(-, Z) \rightarrow \operatorname{hom}_{\Lambda}(-, M)$ defined as hom(id $\left.{ }_{X}, \beta\right)$ for each $X \in M^{0}$. Since the representable functors $\operatorname{hom}_{\Lambda}(-, X)$ are projective in the functor category, $B_{*}$ cannot be an epimorphism and so $\operatorname{Im} \beta_{*}$ must be contained in the unique maximal subfunctor $J(-, M)$ of $\operatorname{hom}_{\Lambda}(-, M)$ [7]. Thus we obtain the commutative diagram

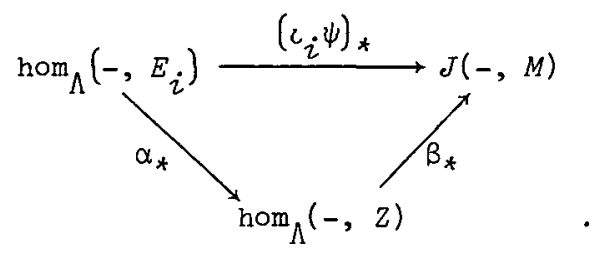

Since $\operatorname{hom}_{\Lambda}(-, E)$ was chosen as a projective cover of $J(-, M)[7]$, we can complete the above diagram in an obvious way to the following one

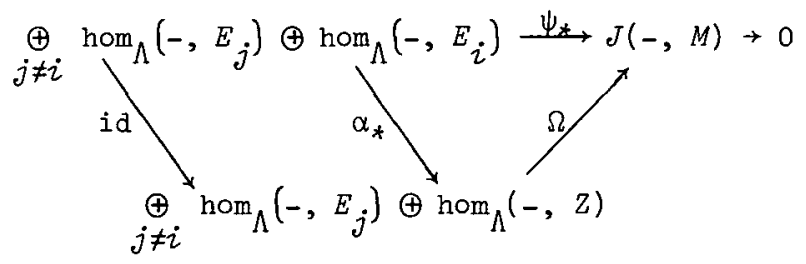

As $\underset{j \neq i}{\oplus} \operatorname{hom}_{\Lambda}\left(-, E_{j}\right) \oplus \operatorname{hom}_{\Lambda}(-, z)$ are projective, we are able to represent $\Omega$ as $\Phi \psi_{*}$ for a suitable $\Phi$. This gives rise to (id $\left.\oplus \alpha_{*}\right) \Phi \psi_{*}=\psi_{*}$. Since $\psi_{*}$ is an essential epimorphism, we conclude that (id $\oplus \alpha_{*}$ ) $\Phi$ is an epimorphism and thus a splittable epimorphism. Going back from representable functors to lattices, one easily verifies now that $\alpha$ is a splittable monomorphism. The second part of $(b)$ is proven similarly. // We now point out a useful fact.

LEMMA 1.4. Let $0 \rightarrow N \stackrel{\varphi}{\longrightarrow} E \stackrel{\Psi}{\longrightarrow} M \rightarrow 0$ be an almost split sequence with $E=E_{1} \oplus E_{2}, E_{i} \neq 0$. Then for $i \neq j$ :

(a) $\varphi \pi_{i}$ is an epimorphism if and only if ${ }^{\prime}{ }_{j} \psi$ is an epimorphism; 
(b) $\varphi \pi_{i}$ is a monomorphism if and only if $\iota_{j} \psi$ is a monomorphism.

Moreover, the epimorphisms in (a), respectively the monomorphisms in (b), have isomorphic kernels, respectively cokernels.

Proof. We content ourselves $w / 1$ th showing part (a). Let $\varphi \pi_{1}$ be a monomorphism. Then the serpent lemma gives rise to the following commutative diagram, which completes the proof:

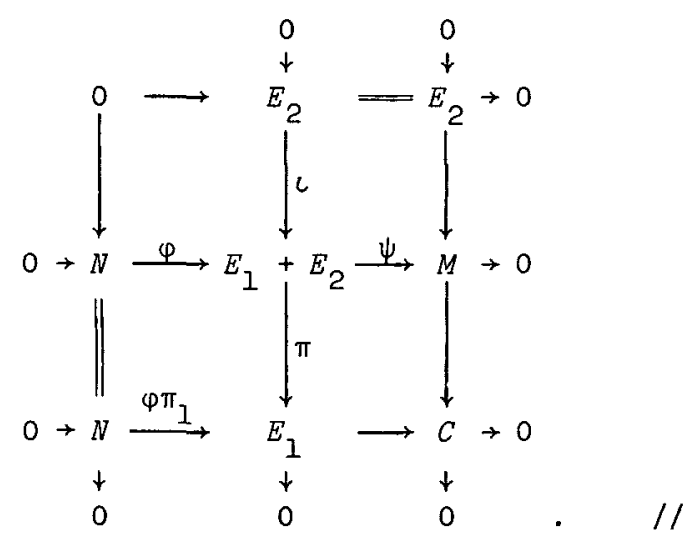

Next we study how often a fixed lattice can occur in the middle term of an almost split sequence.

PROPOSITION 1.5. Let $X \in M^{0}$. Then there are only finitely many almost split sequences $0 \rightarrow N \rightarrow E \rightarrow M \rightarrow 0$ such that $X$ is isomorphic to a direct summond of $E$.

Proof. Without loss of generality we can assume that $X$ is indecomposable. If $X$ is projective, then the irreducible morphism from $N$ to $X$ must be a monomorphism, which restricts $N$ to finitely many possibilities by the Jordan-Zassenhaus Theorem [8]. On the other hand, if $X$ is not projective, we have an almost split sequence $0 \rightarrow Z \rightarrow Y \rightarrow X \rightarrow 0$. Thus Theorem 1.3 implies that $N$ is isomorphic to a direct summand of $Y$. / /

This result gives rise to the question of what it means that the midale term of an almost split sequence $0 \rightarrow N \rightarrow E \rightarrow M \rightarrow 0$ has a projective direct summand. An answer is given by 


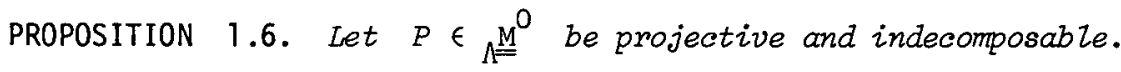

(a) If $\varphi: N \rightarrow P$ is irreducible, then $N$ is isomorphic to a direct summand of the radical of $P, \operatorname{rad}(P)$.

(b) If $X$ is a direct summand of $\operatorname{rad}(P)$, then the inclusion $\iota: X \rightarrow P$ is irreducible.

Proof. (a) Since $\varphi$ is not an epimorphism, Im $\varphi$ must be contained in the unique maximal submodule $\operatorname{rad}(P)$ :

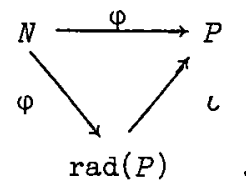

$\varphi$ being irreducible, this implies $N \mid \operatorname{rad}(P)$. (The symbol | means 'isomorphic to a direct summand'.)

(b) Let $\iota_{1}: X \rightarrow \operatorname{rad}(P) \operatorname{resp} \pi_{1}: \operatorname{rad}(P) \rightarrow X$ be the injection, respectively projection, and $\iota: \operatorname{rad}(P) \rightarrow P$ be the inclusion. Assume that there is a factorization via $Y: \rho n=\iota_{1} \iota$. This gives the commutative diagram

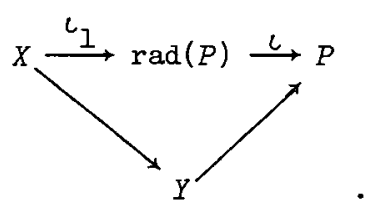

If $\eta$ is not a splittable epimorphism, $\eta$ factors via $\operatorname{rad}(P): \eta=\mu c$. Thus $\rho \mu \iota=\iota_{1} \iota$, which implies $\rho \mu=\iota_{1}$, since $\iota$ is a monomorphism. So we obtain $\rho \mu \pi_{1}=\iota_{1} \pi_{1}=i d_{X}$, and $\rho$ is a splittable monomorphism. $/ /$

Let us denote by $0 \rightarrow N_{i} \rightarrow E_{i} \rightarrow M_{i} \rightarrow 0$ those finitely many almost split sequences with $N_{i} \mid \operatorname{rad}(P)$ for some indecomposable projective $P \in M^{\mathrm{M}}$. Then the set $\underline{\underline{U}}=\left\{M_{i}\right\}$ is characterized by the following property.

THEOREM 1.7. If $X \in \Lambda^{\mathrm{M}}=$ is non-projective and indecomposable such 
that, given any epimorphism $\alpha: Y \rightarrow X$, then $\alpha$ splits or $Y$ has a projective direct sumand, then $X \in \underline{\underline{U}}$ and conversely each $X \in \underline{\underline{U}}$ has this property.

Proof. By Proposition 1.6 we only have to show one direction. Let $M_{i} \in \underline{\underline{U}}$; that is, $0 \rightarrow N_{i} \stackrel{\varphi}{\rightarrow} E_{i} \stackrel{\psi}{\longrightarrow} M_{i} \rightarrow 0$ is an almost split sequence with $N_{i} \mid \operatorname{rad}(P)$ and therefore $P \mid E_{i}$. Assume that $\alpha: Y \rightarrow M_{i}$ is an epimorphism, which does not split. Since $P \mid E_{i}$ is projective, we can complete the following diagram by $B: P \rightarrow Y:$

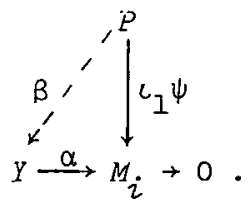

As $c_{1} \psi$ is irreducible, we conclude that $\beta$ is a splittable monomorphism. This implies $P \mid Y$. //

\section{Almost split sequences for Gorenstein orders}

From now on let $\Lambda$ be a Gorenstein order; that is, $\Lambda^{*}=\operatorname{hom}_{R}(\Lambda, R)$ is a progenerator for $\stackrel{M}{M}$ and $\underline{M}_{\Lambda}^{O}$. Note that in this case the projective and relatively injective lattices coincide. The most important example of a Gorenstein order is given by the group ring $R G$ for a finite group $G$.

We restate the following theorem from [6].

THEOREM 2.1. Let $0 \rightarrow K \rightarrow P \rightarrow M \rightarrow 0$ be a projective cover sequence for $M$ and $0 \rightarrow N \rightarrow E \rightarrow M \rightarrow 0$ an almost split sequence then $N \cong K$. Moreover let $0 \rightarrow N^{\prime} \rightarrow Q \rightarrow N \rightarrow 0$ be a projective cover sequence for $N$. Then in the following commutative diagram the first row is an almost split sequence: 


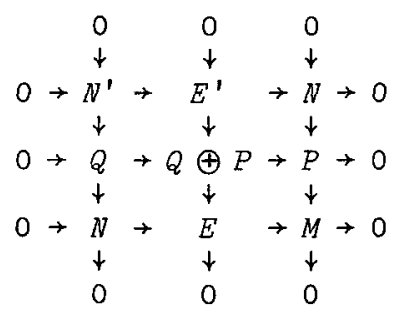

We point out what it means if the middle term of an almast split sequence has a proper direct summand which is projective.

THEOREM 2.2. Let $0 \rightarrow N \stackrel{\varphi}{\longrightarrow} E \oplus P \stackrel{\psi}{\rightarrow} M \rightarrow 0$ be an almost split sequence with $E, P \neq 0$ and $P$ projective. Then $E, P, N$, and $M$ have the same rank and $P$ is indecomposable. Moreover $\operatorname{rad}(P)$ and $\operatorname{rad}^{-}(P)$, the unique minimal overmodule of $P$, are indecomposable.

Proof. If we denote by $\pi_{2}$ the projection from $E \oplus P$ to $P$, then it is clear that $\varphi \pi_{2}$ is irreducible and so it is a monomorphism, since otherwise it would split. Using Proposition 1.2 (c) we have only the possibilities that $\varphi \pi_{2}$ is a monomorphism onto a maximal submodule of $P$, or $\varphi \pi_{2}$ is a pure monomorphism. Assuming the last one, we obtain an exact sequence of lattices

$$
0 \rightarrow N \stackrel{\varphi \pi_{2}}{\rightarrow} P \rightarrow C \rightarrow 0
$$

and so $0 \rightarrow C^{*} \rightarrow P^{*} \rightarrow N^{*} \rightarrow 0$ is exact, too. Because of Proposition 1.2 (c), $C^{*}$ is indecomposable, which implies that $P^{*}$ is isomorphic to the projective cover $P\left(N^{*}\right)$ on $N^{*}$. On the other hand $0 \rightarrow M^{*} \rightarrow E^{*} \oplus P^{*} \rightarrow N^{*} \rightarrow 0$ is an almost split sequence, again. Thus by Theorem 2.1 it follows that $\mathrm{rk}\left(E^{*} \oplus P^{*}\right)=\mathrm{rk}\left(P^{*}\right)$, a contradiction to $E \neq 0$. So $\varphi \pi_{2}$ is a monomorphism onto a maximal submodule of $P$. Using the same arguments one shows that $\iota_{2} \psi \in$ hom $_{\Lambda}(P, M)$ cannot be an epimorphism, so it must be a monomorphism. Since $P$ is relatively injective, $\iota_{2} \psi$ has to be a monomorphism onto a maximal submodule of $M$, otherwise it would split. So, by Lemma 1.4, we have shown that $N, E, P, M$ have the same rank. Suppose now that $P=P_{1} \oplus P_{2}$ is a decomposition with $P_{i} \neq 0$. Then we 
apply our result to $E^{\prime}=E \oplus P_{1}$ and $P^{\prime}=P_{2}$ to obtain $\operatorname{rk}(E)+\operatorname{rk}\left(P_{1}\right)=\operatorname{rk}\left(P_{2}\right)$, a contradiction to $\operatorname{rk}(E)=\operatorname{rk}\left(P_{1}\right)+\operatorname{rk}\left(P_{2}\right)$. The rest of the proof follows from Proposition 1.6. //

REMARK 2.3. (a) This gives a complete description of all almost split sequences 'with projectives'. As a matter of fact either $\operatorname{rad}(P)$ is indecomposable and Theorem 2.2 occurs or $\operatorname{rad}(P)$ decomposes to $\underset{i=1}{\oplus} N_{i}$ and then the exact sequences $0 \rightarrow N_{i} \stackrel{\varphi_{i}}{\longrightarrow} P \rightarrow \operatorname{cok} \varphi_{i} \rightarrow 0$ are almost split, since $\operatorname{cok} \varphi_{i}$ is indecomposable and in the almost split sequence $0 \rightarrow K_{i} \rightarrow E_{i} \rightarrow \operatorname{cok} \varphi_{i} \rightarrow 0$ there is $K_{i} \simeq N_{i}$ by Theorem 2.1 and $E_{i} \simeq P$ by Theorem 1.3 .

(b) Wé have another obvious consequence of the above result. If we denote by $n_{0}$ the maximal rank of the indecomposable projective lattices, then for each indecomposable $M \in \Lambda_{\Lambda} \stackrel{M}{0}^{0}$ with $\operatorname{rk}(M)>n_{0}$ the middle term of those almost split sequences which have $M$ on the left, respectively right, side are not projective and do not have projective direct summands.

The following fact says that those almost split sequences which coincide with projective cover sequences, are in some sense isolated.

PROPOSITION 2.4. Let $0 \rightarrow N \rightarrow E \rightarrow M \rightarrow 0$ be an almost split sequence. Then $E$ is projective if and only if $N$ and $M$ do not occur in any almost split sequence as a direct sumand of the middle term.

Proof. Let $E$ be projective and suppose that $0 \rightarrow X \rightarrow Y \rightarrow Z \rightarrow 0$ is an almost split sequence. If $N \mid Y$, then $N \rightarrow Z$ is irreducible by Theorem 1.3 and so $z \mid E$. This would imply that $z$ is projective, a contradiction. The rest is proven similarly. //

Now we turn to group rings $R G$, where $G$ is a finite group with order not equal to 1 . Let

$$
\cdots P_{2} \stackrel{\varphi_{2}}{\longrightarrow} P_{1} \stackrel{\varphi_{1}}{\longrightarrow} P_{0} \stackrel{\varphi_{0}}{\longrightarrow} R \rightarrow 0
$$

be a minimal projective resolution of $R$. Then the syzygies $\Omega_{i}$ of $R$ 
are defined as $\Omega_{i}=\operatorname{ker} \varphi_{i}, i=0,1,2, \ldots$. For technical reasons we put $\Omega_{-1}=R$.

We have the following result concerning almost split sequences for syzygies .

THEOREM 2.5. Let $R G$ be indecomposable and $0 \rightarrow \Omega_{i} \rightarrow E_{i} \rightarrow \Omega_{i-1} \rightarrow 0$ be an almost split sequence for $i=0,1, \ldots$.

(a) $E_{i}$ is indecomposable.

(b) $R$ is periodic if and only if there exist only finitely many non-isomorphic $E_{i}$.

(c) If any $E_{i}$ is projective, then $R$ is periodic.

Proof. (a) First we prove that there is no $E_{i}$ which has a projective proper direct summand. Since $R G$ is indecomposable, we can put $\operatorname{rk}\left(P_{j}\right)=n_{j}|G|$ for some integers $n_{j}$. Thus we obtain, by (1), the following formula

$$
\operatorname{rk}\left(\Omega_{i}\right)=n_{i}|G|-n_{i-1}|G|+n_{i-2}|G| \ldots \pm I .
$$

Supposing now that $E_{i}$ has a projective proper direct summand $P$; Theorem 2.2 implies $\operatorname{rk}(P)=\operatorname{rk}\left(\Omega_{i}\right)=\operatorname{rk}\left(\Omega_{i+1}\right)$ and $P$ is indecomposable. Thus $|G|=2|G|+1=m|G|-1$ for some integers $l, m$. This is a contradiction, since $|G| \neq 1$ by assumption.

Now we show by induction that $E_{i}$ is indecomposable. Suppose there is a non-trivial decomposition $E_{0}=E \oplus E^{\prime}$. Since $\Omega_{0}$ is the augmentation ideal $\underline{\underline{\mathrm{E}}}$, we have $\operatorname{hom}_{R G}\left(\Omega_{0}, R\right)=0$. This implies that neither $E$ nor $E^{\prime}$ are isomorphic to $R$. Thus in the almost split sequence $0 \rightarrow \Omega_{0} \stackrel{\psi}{\longrightarrow} E \oplus E^{\prime} \stackrel{\varphi}{\longrightarrow} R \rightarrow 0$ the irreducible homomorphisms $\iota_{1} \varphi$ and $\iota_{2}^{\varphi}$ are epimorphisms by Proposition 1.2. Using Lemma 1.4 we conclude that $\psi \pi_{1}$ and $\psi \pi_{2}$ are epimorphisms, too. So we obtain the epimorphism $\psi \pi_{1} c_{1} \varphi$ from $\Omega_{0}$ onto $R$, a contradiction to hom $_{R G}\left(\Omega_{0}, R\right)=0$. 
Assume now that $E_{i}$ is indecomposable for all $i \leq n$. We have the commutative diagram, where $\underline{E}_{n}$ and $E_{n+1}$ are almost split sequences

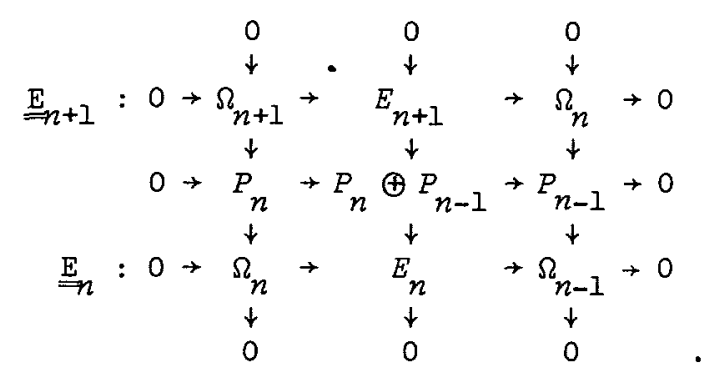

If $E_{n}$ is projective, then $E_{n+1}$ is projective, too, and since $E_{n+1}$ can not have a projective proper direct summand, $E_{n+1}$ is indecomposable. If $E_{n}$ is not projective, then $E_{n+1}$ has no projective direct summand and so the exact sequence $0 \rightarrow E_{n+1} \rightarrow P_{n} \oplus P_{n-1} \rightarrow E_{n} \rightarrow 0$ is a projective cover sequence, which implies $E_{n+1}$ to be indecomposable.

(b) Assume that $R$ is not periodic. Since each indecomposable module occurs as a middle term in only finitely many almost split sequences by Proposition 1.5, there must exist infinitely many non-isomorphic $E_{i}$. The converse is trivial.

(c) This is a special case of (b). In fact, if $E_{n}$ is projective, then $E_{j}$ is projective and indecomposable for all $j \geq n$ and so there occur only finitely many non-isomorphic $E_{i}$. //

If we exclude the case where $E_{0}$ is projective - by Proposition 1.6 this can only happen if $R \oplus \underline{\underline{\mathrm{g}}} \simeq \operatorname{rad}(R G)$ - all the $E_{i}$ are non-projective indecomposable, and so there exist almost split sequences for $E_{i}$.

PROPOSITION 2.6. There exists an $X_{i} \neq 0$ such that $0 \rightarrow E_{i+1} \rightarrow \Omega_{i} \oplus X_{i} \rightarrow E_{i} \rightarrow 0$ is an almost split sequence.

Proof. From Theorem 2.5 we get the almost split sequences

(1) $0 \rightarrow \Omega_{i+1} \stackrel{\alpha}{\longrightarrow} E_{i+1} \stackrel{\beta}{\longrightarrow} \Omega_{i} \rightarrow 0$, 
(2) $0 \rightarrow \Omega_{i} \stackrel{\gamma}{\rightarrow} E_{i} \stackrel{\delta}{\longrightarrow} \Omega_{i-1} \rightarrow 0$.

If $0 \rightarrow E_{i+1} \rightarrow Y \rightarrow Z \rightarrow 0$ is another almost split sequence, then (1) implies $\Omega_{i} \mid Y$, since $\beta: E_{i+1} \rightarrow \Omega_{i}$ is irreducible. So there is an irreducible morphism from $\Omega_{i}$ to $Z$, which means $Z \simeq E_{i}$. Thus there is ar. $X_{i}$ such that

$$
0 \rightarrow E_{i+1} \rightarrow \Omega_{i} \oplus X_{i} \rightarrow E_{i} \rightarrow 0
$$

is an almost split sequence. Since $\operatorname{rk}\left(E_{j}\right)=k_{j}|G|$ and $\operatorname{rk}\left(\Omega_{j}\right)=\tau_{j}|G| \pm 1$, we obtain $\operatorname{rk}\left(X_{i}\right)=m|G| \pm 1$ for some integer $m$. This implies $X \neq 0$, because $G$ was chosen non-trivial. //

It would be interesting to know more about that lattice $X_{i}$.

\section{Examp 1e}

Let $p$ be a prime, $G$ the cyclic group of order $p^{2}, R=\mathbb{Z}_{p}$, and $\Lambda=R G$. From [4,5] we know that $\Lambda$ has $4 p+1$ indecomposable nonisomorphic lattices. We want to apply some of our previous results to get information about almost split sequences for lattices over $\Lambda$. Since it is more comfortable to work with a concrete number, we put $p=5$ (without loss of generality). Then the ranks of the indecomposable lattices are:

$\begin{array}{cc}\text { rank } & \begin{array}{c}\text { number } \\ 26\end{array} \\ 25 & \text { indecomposable } \\ 24 & 3 \\ 21 & 9 \\ 20 & 4 \\ 5 & 1 \\ 4 & 1 \\ 1 & 1 \\ & 1 \\ & 1\end{array}$

We start with the almost split sequence for $R$. From [7] we know that this sequence is obtained from the following commutative diagram: 


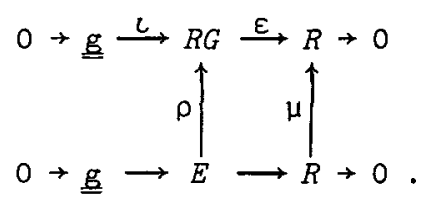

Here $\varepsilon$ is the augmentation map, $\underline{\underline{g}}$ is the augmentation ideal, and $\mu$ multiplication by $p$. Now cok $\mu$ is a simple torsion module, so the serpent lemma implies that cok $\rho$ is simple, too. From this we conclude that $E$ is isomorphic to $\operatorname{rad}(R G)$, since $\operatorname{rad}(R G)$ is the unique maximal submodule of $R G, R G$ being indecomposable. As an application of Theorem 2.5 we obtain

$$
\operatorname{rad}(R G) \text { is indecomposable. }
$$

So, by Theorem 2.2,

$$
0 \rightarrow E \rightarrow R G \oplus Y^{\prime} \rightarrow Z \rightarrow 0
$$

is the only almost split sequence, which has $R G$ as a direct summand of the middle term, and $\operatorname{rk}\left(Y^{\prime}\right)=\operatorname{rk}(Z)=25$.

Next we are interested in the almost split sequence for $\underline{g}$. Since $G$ is cyclic, $\underline{g}$ is cyclic as $R G$-module and we obtain the projective cover sequence $0 \rightarrow R \rightarrow R G \rightarrow \underline{\mathrm{g}} \rightarrow 0$ and the almost split sequence

$$
0 \rightarrow R \rightarrow F \rightarrow \underline{\underline{\mathrm{g}}} \rightarrow 0 \text {. }
$$

Theorem 2.2 implies that $F$ is indecomposable and Proposition 2.6, together with (1), gives rise to the almost split sequences

$$
0 \rightarrow R \rightarrow \underline{\underline{g}} \oplus X \rightarrow E \rightarrow 0, \quad \operatorname{rk}(X)=26
$$

and

$$
0 \rightarrow E \stackrel{\alpha}{\longrightarrow} R \oplus R G \oplus Y \stackrel{\beta}{\longrightarrow} F \rightarrow 0, \quad \operatorname{rk}(Y)=24 .
$$

Since $\alpha \pi$ and $\iota B$ are irreducible for each direct summand of $R \oplus R G \oplus Y$ and so the kernel, respectively cokernel, of $\alpha \pi$ and $C B$ are indecomposable by Proposition 1.2, we get information about $Y$. For example if $Y$ has a direct summand $Y_{1}$ of rank 5 , then for $\pi: R \oplus R G \oplus Y \rightarrow R \oplus R G \oplus Y_{1}, \quad r k(\operatorname{cok} \alpha \pi)=31-25=6$, a contradiction. By similar arguments we conclude that there are only two possibilities for $Y ; Y$ indecomposable, or $Y=Y_{1} \oplus Y_{2}$ with $\operatorname{rk}\left(Y_{1}\right)=20, \operatorname{rk}\left(Y_{2}\right)=4$, 
and for $X: X$ indecomposable or $X=X_{1} \oplus X_{2}$ with $\operatorname{rk}\left(X_{1}\right)=21$, $r k\left(X_{2}\right)=5$. Moreover, it is easy to show that $X$ is indecomposable if and only if $Y$ is indecomposable. In fact, assume that $0 \rightarrow F \rightarrow \underline{\underline{g}} \oplus X_{1} \oplus X_{2} \rightarrow E \rightarrow 0$ is almost split. Then $0 \rightarrow X_{2} \rightarrow E$ is irreducible and so $0 \rightarrow X_{2} \rightarrow E \rightarrow C \rightarrow 0$ is almost split. Then $C$ has rank 20 and since $E \rightarrow C \rightarrow 0$ is irreducible, Theorem 1.3 implies $C \mid Y$. The converse is proven similarly. In both cases, Theorem 1.3 gives much information about other almost split sequences. For example, if $X=X_{1} \oplus X_{2}$, then we obtain together with (4) and (5), that the following sequences are almost split:

$$
\begin{aligned}
& 0 \rightarrow X_{1} \rightarrow E \rightarrow Y_{2} \rightarrow 0, \\
& 0 \rightarrow Y_{1} \rightarrow E \rightarrow X_{2} \rightarrow 0, \\
& 0 \rightarrow X_{2} \rightarrow E \rightarrow Y_{1} \rightarrow 0,
\end{aligned}
$$

and

$$
0 \rightarrow Y_{2} \rightarrow F \rightarrow X_{1}+0
$$

Finally we study the almost split sequences for those lattices $M$ which have rank 26 . Let $0 \rightarrow N \rightarrow R G^{(k)} \rightarrow M \rightarrow 0$ be a projective cover sequence. Then $N$ is indecomposable and so $k=2$, since otherwise there would exist an indecomposable lattice with rank greater than 26 . This implies $\operatorname{rk}(N)=24$ but $N \neq \underline{\mathrm{g}}$, because there is a projective cover sequence $0 \rightarrow \underline{\mathrm{g}} \rightarrow R G \rightarrow R \rightarrow 0$. Let

$$
0 \rightarrow N \stackrel{\varphi}{\longrightarrow} U \stackrel{\Psi}{\longrightarrow} M \rightarrow 0
$$

be almost split. Then $R$ is not a direct summand of $U$ since otherwise $\operatorname{rk}(\operatorname{ker} \varphi \pi)=24-1=23$, a contradiction. Similarly one shows $U=U_{1} \oplus U_{2}$ with $U_{i}$ indecomposable, $\operatorname{rk}\left(U_{i}\right)=25$, and $U_{k} \neq R G$ by Theorem 2.2. 


\section{References}

[1] Maurice Auslander, "Almost split sequences I", Representations of algebras, 1-8 (Proc. Internat. Conference, Ottawa, 1974. Lecture Notes in Mathematics, 488. Springer-Verlag, Berlin, Heidelberg, New York, 1975).

[2] Maurice Auslander, "Existence theorems for almost split sequences", Comm. Azgebra (to appear).

[3] Maurice Auslander and Idum Reiten, "Almost split sequences II", Representations of algebras, 8-19 (Proc. Internat. Conf., Ottawa, 1974. Lecture Notes in Mathematics, 488. Springer-Verlag, Berlin, Heidelberg, New York, 1975).

[4] A. Heller and 1. Reiner, "Representations of cyclic groups in rings of integers, I", Ann. of Math. (2) 76 (1962), 73-92.

[5] A. Heller and 1. Reiner, "Representations of cyclic groups in rings of integers, II", Ann. of Math. (2) 77 (1963), 318-328.

[6] K.W. Roggenkamp, "Almost split sequences for group rings", Mitt. Math. Sem. Giessen 121 (1976),

[7] K.W. Roggenkamp and J.W. Schmidt, "Almost split sequences for integral group rings and orders", Comm. AZgebra 4 (1976), 893-917.

[8] Hans Zassenhaus, "Neuer Beweis der Endlichkeit der Klassenzahl bei unimodularer Äquivalenz endlicher ganzzahliger Substitutionsgruppen", Abh. Math. Sem. Univ. Hamburg 12 (1938), 276-288.

Mathematisches Institut der Universität,

Stuttgart,

Bundesrepublik Deutschland. 